\title{
The role of the myosin ATPase activity in adaptive thermogenesis by skeletal muscle
}

\author{
Roger Cooke
}

Received: 18 November 2010 / Accepted: 4 February 2011 /Published online: 23 March 2011

(C) The Author(s) 2011. This article is published with open access at Springerlink.com

\begin{abstract}
Resting skeletal muscle is a major contributor to adaptive thermogenesis, i.e., the thermogenesis that changes in response to exposure to cold or to overfeeding. The identification of the "furnace" that is responsible for increased heat generation in resting muscle has been the subject of a number of investigations. A new state of myosin, the super relaxed state (SRX), with a very slow ATP turnover rate has recently been observed in skeletal muscle (Stewart et al. in Proc Natl Acad Sci USA 107:430-435, 2010). Inhibition of the myosin ATPase activity in the SRX was suggested to be caused by binding of the myosin head to the core of the thick filament in a structural motif identified earlier by electron microscopy. To be compatible with the basal metabolic rate observed in vivo for resting muscle, most myosin heads would have to be in the SRX. Modulation of the population of this state, relative to the normal relaxed state, was proposed to be a major contributor to adaptive thermogenesis in resting muscle. Transfer of only $20 \%$ of myosin heads from the SRX into the normal relaxed state would cause muscle thermogenesis to double. Phosphorylation of the myosin regulatory light chain was shown to transfer myosin heads from the SRX into the relaxed state, which would increase thermogenesis. In particular, thermogenesis by myosin has been proposed to play a role in the dissipation of calories during overfeeding. Up-regulation of muscle thermogenesis by pharmaceuticals that target the SRX would provide new approaches to the treatment of obesity or high blood sugar levels.
\end{abstract}

R. Cooke $(\bowtie)$

Department of Biochemistry \& Biophysics,

Cardiovascular Research Institute, University of California,

Box 2240, Genentech Hall, 600, 6th Street,

San Francisco, CA 94158-2517, USA

e-mail: cooke@cgl.ucsf.edu
Keywords Myosin - Adaptive thermogenesis - Obesity · Skeletal muscle $\cdot$ Fluorescence $\cdot$ Metabolic rate

\section{Introduction}

The existence of a new state of myosin with a very low ATPase activity, the super relaxed state (SRX) has a variety of consequences for interpreting existing data on myosin function (Stewart et al. 2010). In particular, the SRX has been proposed to play a role in adaptive thermogenesis by resting muscle. The goal of the present review is to first briefly review the data supporting the existence of the new state. Thermogenesis by resting muscle and its role in whole body metabolism is considered. I then describe how the new state of myosin could provide a mechanism for thermogenesis and integrate this proposed mechanism into our current knowledge of muscle thermogenesis. I specifically discuss how the SRX could play a role in the response to overfeeding. Two fields of research into muscle function, one concerned with the contractile proteins as force generators and one concerned with the role of muscle in adaptive thermogenesis, should now find common ground. These two fields have previously had little interaction, and it is the goal of this review to try to bridge that gap.

\section{The ATPase activity of purified myosin is not compatible with the metabolic rate of resting muscle}

The presence of an inhibited state of skeletal myosin in resting muscle was first suggested more than 30 years ago when the ATPase activity of purified myosin was compared for the first time with the resting metabolic rate of the muscle from which it came (Ferenczi et al. 1978). Although 
the myosin ATPase activity was low, $0.011 \mathrm{~s}^{-1}$, the heat produced by this activity alone would be greater than that observed for the living muscle by about a factor of five (Ferenczi et al. 1978; Kushmerick and Paul 1976). This was clear evidence that myosin must be inhibited in vivo by a mechanism that at that time was unknown. The degree of inhibition must be considerably greater than the factor of five, as the resting metabolism of skeletal muscle is low and, in addition to the metabolism of myosin, ATPase must also carry out other obligatory cell functions, such as protein synthesis, ion pumping, etc. A similar argument was made for rabbit myosin in comparison to living resting rabbit muscle (Gutierrez et al. 1989; Myburgh et al. 1995).

The data discussed above lead unambiguously to the conclusion that in living resting muscle the ATPase activity of myosin must be inhibited to a level below that of isolated myosin itself. The existence of a new state of myosin is of interest in itself, as it is likely to play a role in muscle function. However, if the population of this state is regulated, with some myosins in the inhibited state while others are in a state with an ATPase activity similar to that of purified myosin, redistribution of myosin between the two states would have a large effect on resting muscle metabolism. As discussed below, the metabolism of resting muscle is variable, and this variability plays a significant role in determining whole body metabolic rate and can, consequently, influence important aspects of human health, such as obesity. To investigate this possibility, several years ago a search was initiated to find an in vitro system in which skeletal myosin could be observed in the inhibited state.

\section{Measuring single nucleotide turnovers in permeable muscle fibers}

Previous studies of in vitro muscle systems had failed to observe a myosin state with an ATPase activity that was sufficiently low to be compatible with living muscle. Several in vitro muscle systems have been extensively studied, including myofibrils and permeable muscle fibers. These preparations contain other proteins, in addition to myosin, with more rapid ATPase activities. A small inhibition of the ATP turnover times of myosin in myofibrils relative to purified myosin was observed, approximately a factor of two, which is in the right direction, but insufficient to explain the above discrepancy (Myburgh et al. 1995). One method for observing a slow ATP turnover in the presence of other faster enzymes is to measure single nucleotide turnovers using fluorescent nucleotides. The fluorescent ATP analog $2^{\prime}-/ 3^{\prime}-O-$ ( $N^{\prime}$-methylanthraniloyl)-ATP (mantATP) has been shown to bind tightly to myosin (Woodward et al. 1991), with a twofold increase in quantum yield (Cremo et al. 1990), making it an excellent probe for studying myosin kinetics.
A highly inhibited state of myosin in skeletal muscle was first observed by measuring single nucleotide turnovers (Stewart et al. 2010). The ATP turnover rate in relaxed permeable rabbit psoas fibers was measured by first incubating the fibers in mantATP followed by a chase with ATP. The fiber was relaxed in both solutions. During the chase phase, the fluorescence intensity of the fiber decreased as the bound mant nucleotides were released and replaced by ATP (Fig. 1). There was a rapid decay in fluorescence intensity followed by a slow decay. A second experimental approach was to incubate the fiber first in ATP, followed by a chase with mantATP, also shown in Fig. 1. The intensity rose as ATP was released and replaced by mantATP. The change in fluorescence occurred in two phases that mirrored those of the first experiment, showing that the mantATP turnover rate was similar to the ATP turnover rate. The rapid phase, which has a time constant of approximately $20 \mathrm{~s}$, comprises multiple factors, including the release of non-specifically bound nucleotides, the release of nucleotides by a fraction of the normally relaxed myosin heads, and the diffusion of released nucleotides out of the fibers, requiring approximately $10 \mathrm{~s}$ (Cooke and Pate 1985). The time constant for the second phase is much

$\begin{array}{cc}\text { INCUBATION } & \text { CHASE } \\ 4 \mathrm{mM} \text { ATP } & 250 \mu \mathrm{M} \text { mATP } \\ 250 \mu \mathrm{M} \text { mATP } & 4 \mathrm{mM} \text { ATP }\end{array}$

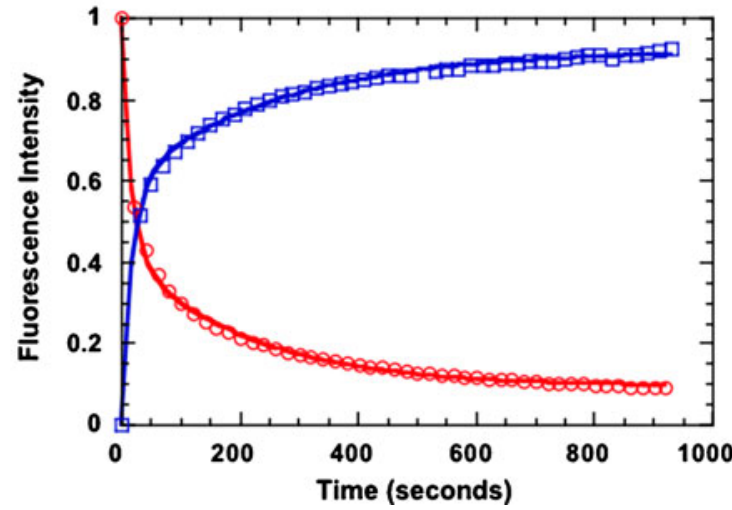

Fig. 1 Fluorescence intensities are shown as a function of time during the chase phase of two single-nucleotide turnover experiments in permeable rabbit fast-twitch muscle fibers. The fluorescence decay occurring during a chase with ATP following an incubation with mantATP [2'-/3'-O-( $N^{\prime}$-methylanthraniloyl)-ATP] is shown (red, open circles). The rise in fluorescence intensity occurring during the inverse experiment in which the fiber was first incubated with ATP followed by a chase with mantATP is also shown (blue, open squares). The composition of the incubation and chase phases are shown above, color-coded with the data. The fluorescence changes in two phases, a rapid phase which has a time constant of approximately $20 \mathrm{~s}$ followed by a slow phase with a time constant of $230 \mathrm{~s}$. The slower phase is attributed to the slow release of nucleotides from a fraction of myosin heads, which are in a "super relaxed" state. Reproduced with permission from (Stewart et al. 2010) 
slower, $230 \mathrm{~s}$, and as discussed below, it arises from the release of nucleotides by a second fraction of myosin heads with a very slow ATP turnover. Although the data described above were obtained from rabbit fast twitch psoas fibers, Stewart et. al. also showed that a similar SRX could be observed in slow twitch rabbit soleus fibers, with a slightly shorter time constant of $156 \mathrm{~s}$ (Stewart et al. 2010).

A number of observations led to the conclusion that the slow phase of fluorescence change, shown in Fig. 1, arose from the slow turnover of nucleotides by myosin heads in the relaxed fibers. The magnitude of the fluorescence of the mant nucleotides specifically bound to the fiber was similar to that expected for binding to myosin alone. The slow release was only observed if the fiber was relaxed, and myosin is the only nucleotide binding protein that responds to the activation level of the fiber. Images of the fiber during the chase showed bright fluorescently labeled A-bands, which contain myosin. Adjusting for the non-specific fluorescence, $50 \%$ of the myosin heads were estimated to have been in the SRX in the initial incubation in the data shown in Fig. 1.

The conclusions, described above led to the proposal of a three-state model of myosin heads in muscle fibers, namely, active, relaxed and "super relaxed" (Fig. 2) (Stewart et al. 2010). The SRX explains the discrepancy described above between the more rapid ATPase activity of purified myosin and the much slower ATPase activity required to be compatible with the metabolic rate of living fibers (Ferenczi et al. 1978). If all myosin heads were in the SRX in living resting muscle under basal conditions, their ATPase activity would account for only $12 \%$ of the metabolic rate. Thus, one important conclusion from this

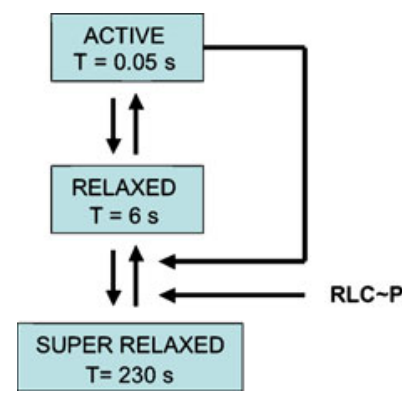

Fig. 2 The three-state model proposed for myosin with approximate nucleotide turnover times. In active muscle, myosin heads are primarily in the active state with rapid nucleotide turnover. In relaxed muscle, myosin can be in one of two states: (1) the normally relaxed state, with a turnover time approximately equal to that of purified myosin, which is $6 \mathrm{~s}$ for rabbit myosin at in vivo temperature or (2) the super relaxed state (SRX), with a much slower turnover time, 230 s. Two factors have been shown to alter rates between the relaxed state and the SRX. Myosin heads in active cycles increase the rate from the SRX to the relaxed state (Fig. 4). Phosphorylation $(\sim P)$ of the regulatory light chain $(R L C)$ also favors the relaxed state, although it is not known whether this is due to increased rates from the SRX to the relaxed state, or to a decreased rate in the reverse direction

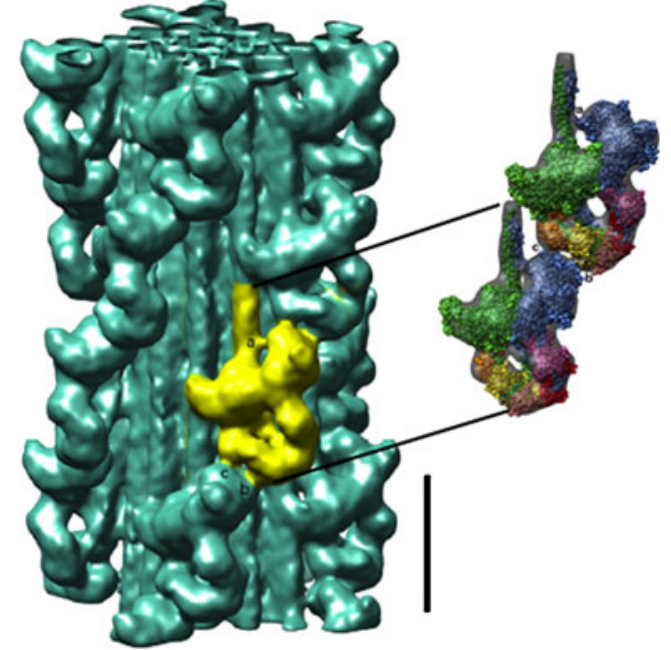

Fig. 3 Three-dimensional reconstruction of the frozen-hydrated tarantula thick filament, filtered to $2-\mathrm{nm}$ resolution. The threedimensional map segment shows four 14.5 crowns of interacting heads. Scale bar: $14.5 \mathrm{~nm}$. Right High-resolution structural models of two relaxed smooth heavy meromyosins in the conserved "J" structural motif (Wendt et al. 1999), which could be fitted, with minor modifications, into the myosin density on the tarantula thick filament. Reproduced with permission from Alamo et al. (2008)

comparison is that under basal conditions, most of the myosin heads must be in the SRX in living fibers.

\section{Structural and biochemical data show myosin ATP turnover is inhibited by the binding of myosin heads to the core of the thick filament}

This highly inhibited state of myosin, described above, was observed in skeletal muscle, a muscle in which regulation occurs by calcium binding to the thin filament. However, myosin with an even slower ATP turnover rate had already been observed in myosin filaments in which regulation occurs via myosin (Vibert and Craig 1985). Inhibition was observed when myosin heads were bound to the core of the thick filament. Early studies of the low-angle diffraction pattern of resting skeletal muscle showed that myosin heads are arranged in a helical array around the core of the thick filament (Huxley et al. 1965). Higher resolution structures of myosin heads in this array were provided by threedimensional reconstructions of electron micrographs of relaxed myosin filaments from tarantula muscle (Fig. 3) (Alamo et al. 2008; Woodhead et al. 2005). The two myosin heads of one molecule interacted with one another in a structural motif resembling a "J". This motif had been seen previously in two-dimensional crystals of unphosphorylated smooth muscle myosin (Wendt et al. 1999). The structure was only seen when myosin was in a relaxed state induced by the binding of ATP and the absence of phosphorylation. A helical array of heads bound to the thick filament was also 
observed at lower resolution in relaxed filaments from scallop muscle, a muscle controlled by binding of calcium to myosin (Al-Khayat et al. 2009; Vibert and Craig 1985).

Myosin heads in the J-motif have a very low ATPase activity. In the absence of ATP filaments became disordered. The time required for disorder to occur during a rigor wash of scallop filaments provided an estimation of the turnover time of scallop myosin, $30 \mathrm{~min}$, showing a remarkable degree of inhibition (Vibert and Craig 1985). A slow release of nucleotides was also seen for smooth muscle myosin in the folded conformation with an ATP turnover time of greater than $50 \mathrm{~min}$, for a non-muscle myosin II and for folded scallop myosin (Ankrett et al. 1991; Cross et al. 1988).

The structural motif seen in tarantula filaments is also found in a variety of other muscles. A similar ordered helical array of myosin heads in the J motif was resolved in myosin filaments from vertebrate cardiac muscle (Zoghbi et al. 2008). Although a high-resolution structure has not been resolved in filaments from skeletal muscle, the structural motif is stabilized by cross-linking and observed in purified monomeric skeletal myosin (Jung et al. 2008). The observation of the folded $\mathrm{J}$ structural motif in a variety of myosins, including those from both thick- and thinfilament-regulated muscles, led Craig and coworkers to propose that the structural motif is conserved across different species and muscle types (Craig and Woodhead 2006; Jung et al. 2008; Zhao et al. 2009). However, the J motif may not be the only motif to be associated with a strong inhibition of the myosin ATPase activity. Filaments in relaxed insect flight muscle show myosin heads in a different structural motif (Al-Khayat et al. 2003). Nonetheless, myosin heads remain in this structural motif for extended times in rigor conditions, showing that the release of nucleotides is slow (Reedy et al. 1992). The inhibition of motor activity by interaction with the tail or rod segments in a folded conformation may be a conserved mechanism for other motors in addition to myosin. A similar inhibited form has been shown to occur in kinesin (Hackney and Stock 2008).

As discussed above, the presence of a structural motif in skeletal myosin, a motif associated with inhibition of ATPase activity, suggested that the inhibition of myosin activity seen in the SRX could also be associated with this motif. This hypothesis was supported by correlations between populations of myosin heads in the ordered helical array and those in the SRX (Stewart et al. 2010). Both the array and the SRX are only observed in relaxed thick filaments, both are increased by higher temperatures, and both are decreased by substitution of GTP for ATP (Kensler and Stewart 1993; $\mathrm{Xu}$ et al. 2003). Phosphorylation of the myosin regulatory light chain (RLC) leads to disorder of skeletal muscle thick filaments seen in electron micrographs (Levine et al. 1996) and also leads to a lower population of myosin heads in the SRX (Stewart et al. 2010). These correlations led to the conclusion that myosin heads in the SRX are bound to the core of the thick filament and have the J motif. This is a parsimonious model that does not invoke a new structure. However, the data are also compatible with a model in which only a subset of the heads bound to the core of the filament are in the SRX (Stewart et al. 2010).

\section{Active fibers}

If myosin heads in the SRX are bound to the core of the thick filament, where they are unable to interact with actin, and their lifetime is long, how is skeletal muscle able to achieve maximal force in a few tens of milliseconds in a tetanic activation? There must be a mechanism in which the activation of the thin filament is sensed by the heads in the SRX, which then leave this state and begin to generate force. Stewart et al. (2010) proposed that the mechanism involved a strong cooperativity between the myosin heads within a thick filament based on their observation that in a chase with ADP all myosin heads left the SRX and quickly turned over their nucleotides. This observation suggests that the interaction of some myosin heads with actin destabilizes the binding of adjacent myosin heads to the core of the thick filament. The structure of the relaxed filaments, shown in Fig. 3, with myosin molecules interacting with adjacent myosin molecules could easily lead to highly cooperative interactions (Alamo et al. 2008). In this model, there must always be some fraction of the myosin heads in the normal relaxed state, which would sense the activation of the thin filament and initiate the cooperative process leading to full activation. Cooperative interactions between myosin heads are also evident in the X-ray diffraction patterns of fibers stretched so that only half of the myosin heads are overlapped by actin (Haselgrove 1975). During activation, all of the myosin heads leave their relaxed positions on the thick filament and become disordered. This indicates that myosin heads interacting with actin in the overlap region rapidly communicate with heads that do not interact with actin.

More recently, more definitive data supporting the hypothesis for fiber activation posed above have been obtained. Fibers relaxed with mantATP were chased with an activating solution (Fig. 4). This resulted in the rapid release of all nucleotides from myosin. Thus, active myosin heads also destabilize myosin heads in the ordered array, as would be expected from the above hypothesis (Fig. 2).

\section{The dynamic nature of the resting metabolic rate of skeletal muscle}

Early investigations of muscle metabolism revealed that the resting metabolic rate of amphibian muscles could vary 


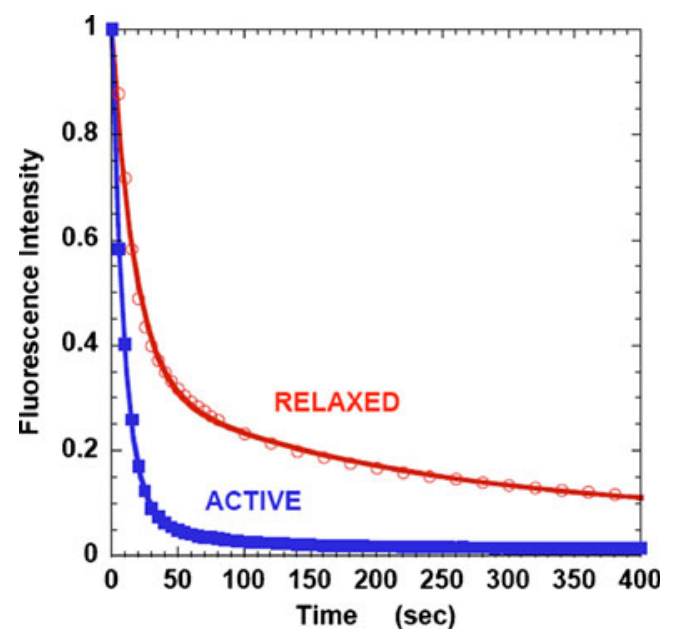

Fig. 4 Fluorescence intensities are shown as a function of time during a chase with a relaxing solution (open circles, red) or with an activating solution (closed squares, blue). In the relaxing solution, there is a slow release of nucleotides from the SRX, while in the activating solution all nucleotides are released in the rapid phase. The fibers were initially relaxed in $250 \mathrm{uM}$ mantATP. The chase solution was either a relaxing solution, containing $4 \mathrm{mM}$ ATP, pCa approx. 9, or an activating solution containing $4 \mathrm{mM}$ ATP and calcium, $\mathrm{pCa}=$ 5.7. The fibers in the activating solution generated $60 \%$ of maximal tension

widely. For example, stretching a muscle to longer lengths could increase metabolism up to fourfold (Feng 1932). The increase occurred over several minutes and was reversible when the muscle was allowed to shorten to its original length (Clinch 1968; Feng 1932). The resting metabolic rate of frog muscle was also increased by higher external concentrations of potassium or by the addition of hyper-osmotic agents in the medium bathing the muscle (Solandt 1936; Yamada 1970).

Hypoxia caused a decrease in the basal metabolic rate of resting frog muscle to $20 \%$ of the control rate and also greatly inhibited the increased thermogenesis seen after stretch and in the presence of higher potassium and hyperosmotic agents (West and Boutilier 1998). The decrease in metabolic rate occurred despite stable ATP concentrations, suggesting that it is under the control of some signaling pathway (West and Boutilier 1998). Prolonged excess metabolism would be deleterious in the absence of oxygen. Subsequent investigations have shown that resting thermogenesis of mouse muscle can also be altered by high external potassium and hyper-osmolality (Chinet 1993; Chinet et al. 1992). During anoxia, resting mouse muscle has an extremely slow ATP turnover rate of $9 \mu \mathrm{M} \mathrm{s}^{-1}$, which would require considerable inhibition of the myosin ATPase activity (Vinnakota et al. 2010). In summary, the resting metabolic rates of both amphibian and mammalian skeletal muscles are highly variable, undergoing large increases or decreases depending on the conditions employed, all of which occur in the absence of active force generation.

\section{Adaptive thermogenesis and the role of muscle}

Whole body energy intake can be broken down into several components (Fig. 5; for review, see Johannsen and Ravussin 2008; Levine 2004; Lowell and Spiegelman 2000; Wijers et al. 2009). An average adult human in our modern society (i. e. not engaged in intense physical activity) consumes approximately $8 \mathrm{MJ} \mathrm{day}^{-1}$. Approximately two-thirds of this energy is used to fuel basic cellular functions, composed of the many exothermic cellular processes required for cell homeostasis, such as protein synthesis, ion pumping, cell division, etc. This component, known as the basal metabolic rate (BMR), does not change by a significant amount. Resting skeletal muscle has a low metabolic rate per unit volume, approximately $0.7 \mathrm{~W} \mathrm{~kg}^{-1}$ in rabbit muscle and $0.5 \mathrm{~W} \mathrm{~kg}^{-1}$ in human muscle. Although these rates are lower than those of many other tissues, such as brain or liver, skeletal muscle contributes about $25 \%$ of the obligatory thermogenesis due its large mass (Zurlo et al. 1990). A proportion of the energy taken in is expended by muscular activity. This component can vary widely depending on one's lifestyle, and for people not involved in manual labor typically amounts to approximately $15-25 \%$ of the total (Levine 2004). There is a component of energy use, known as adaptive thermogenesis, which is altered by conditions, such as cold exposure and the ingestion of excess calories. The difference between the energy taken in and that used by the metabolic components given above is made up for by the storage or consumption of fat and, to a lesser extent, of protein and glycogen. Because the BMR does not change significantly, adaptive thermogenesis and activity both play an important role in maintaining body weight. This review will concentrate on thermogenesis by resting skeletal muscle, its role in adaptive thermogenesis and, in

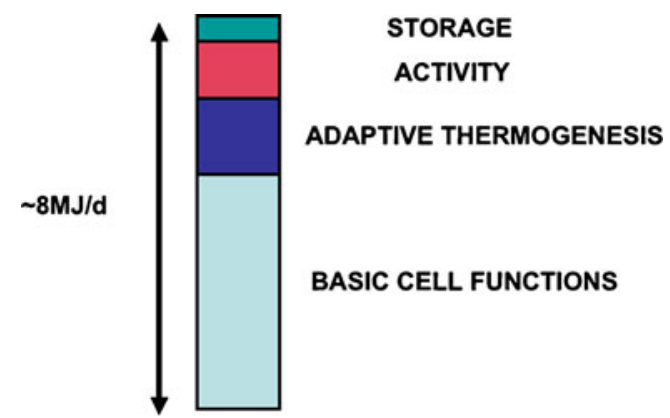

Fig. 5 Components of energy use in the human body. Energy intake in the diet for an average adult human in our modern society (i.e. not engaged in intense physical activity) is approximately $8 \mathrm{MJ} \mathrm{day}^{-1}$. Approximately two-thirds of this energy is required for obligatory cellular functions. A variable amount is used to power physical activity. Adaptive thermogenesis, responding to exposure to cold and to nutritional state, is highly variable and typically amounts to around $25 \%$ of the total. The difference between energy intake and energy used by the factors listed above is either stored to - or retrieved from-lipids, proteins and glycogen. 
particular, on the question of what is the furnace within the muscle responsible for producing the heat. Our group has recently proposed a new mechanism for producing heat in resting muscle, discussed above, and this model will be integrated into the existing physiological data on adaptive thermogenesis and whole body metabolism.

Resting muscle thermogenesis increases following a meal and during exposure to cold (for review, see Johannsen and Ravussin 2008; Lowell and Spiegelman 2000; Wijers et al. 2009). Following a meal, whole body energy expenditure increases by $25-40 \%$ in humans (Sims and Danforth 1987). This is partially due to the energy required for digestion, absorption and storage of nutrients, which is an obligatory thermogenesis, but an additional heat is generated, above that required for digestion. This adaptive thermogenesis is produced by norepinephrine released from sympathetic nerves and by adrenal secretion of epinephrine, and a large portion of this metabolic process, approximately $40 \%$, occurs in resting muscle (Astrup et al. 1986, 1989). Epinephrine has been shown to directly increase muscle thermogenesis (Astrup et al. 1989; Simonsen et al. 1992). Leptin is a hormone released by fat cells that leads to an increased metabolic rate and to reduced satiety. Leptin has also been shown to directly increase resting skeletal muscle thermogenesis (Dulloo et al. 2002). The response to cold exposure is complex and involves both non-shivering and shivering thermogenesis in skeletal muscle. Duchamp and Barre (1993) demonstrated that $70 \%$ of the non-shivering heat generated by ducklings during cold exposure was due to thermogenesis in skeletal muscle. Humans respond to cold exposure by increasing thermogenesis, which is regulated by the central nervous system via sympathetic stimulation (Dauncey 1981; Paolone and Paolone 1995; Simeckova et al. 2000; van Marken Lichtenbelt and Daanen 2003). As discussed above, epinephrine has been shown to activate thermogenesis, showing that skeletal muscle is involved in cold-induced thermogenesis. In summary, resting muscle thermogenesis responds to cold and to food consumption, and epinephrine and leptin have been identified as being involved in some of the pathways controlling this process.

\section{What is the "furnace" responsible for increased thermogenesis in resting skeletal muscle?}

Uncoupling proteins (UCP) are small transmembrane proteins in the inner mitochondrial membrane that can short circuit electron transport, creating a futile cycle that produces heat. The discovery of an UCP that is highly and selectively expressed in skeletal muscle, UCP3, led to the suggestion that this protein may play a dominant role in muscle thermogenesis (for review, see Costford et al. 2007).
However, the results of other studies indicate that UCP3 has a less major role in muscle thermogenesis. The expression of UCP3 was found to increase upon fasting in humans (Gong et al. 2000; Vidal-Puig et al. 2000); this increase in a protein that wastes energy at a time when energy is being conserved undermined the hypothesis that it was a major site of thermogenesis. The over-expression or knockout of UCP3 in mouse muscle had little effect on thermogenesis by resting excised muscles, showing UCP3 did not play a major role in this process (Barclay et al. 2009). The UCP3 knockout mouse is neither obese nor cold intolerant, supporting this suggestion (Gong et al. 2000; Vidal-Puig et al. 2000). However, knockout of UCP1, known to play a role in thermogenesis by brown fat, also does not lead to obesity, although the knockout mouse muscle is cold-intolerant (Enerback et al. 1997). A variety of more recent studies have suggested that the major role of UCP3 in skeletal muscle is in the control of fatty acid metabolism and the mitigation of the deleterious effects of reactive oxygen species (for review, see Costford et al. 2007). The observation that the knockout of UCP1 does not lead to obesity shows that there are mechanisms that can compensate for the lack of thermogenesis. One possible pathway is the one suggested here, involving the modulation of the SRX.

Changes in the rate of thermogenesis of resting muscle have been proposed to arise from increased ion fluxes or futile cycling of lipids. There is evidence that calcium fluxes account for approximately $20-25 \%$ of basal metabolism and can account for a portion of the response to hyperosmotic agents and high external potassium (Chinet 1993; Chinet et al. 1992; Dulloo et al. 1994). Calcium-dependent thermogenesis decreases upon the consumption of fish oil, providing a possible connection to diet (Dulloo et al. 1994). There is no method for directly measuring calcium fluxes, so the above data were obtained by observing the response to agents known to influence calcium flux. However, one agent commonly used in these studies, butanedione-monoxime, is known to also inhibit myosin ATPase activity, leading to an overestimate of the role of thermogenesis by calcium fluxes. Fluxes of sodium and potassium across the cell membrane have been shown to account for only $5 \%$ of resting muscle thermogenesis (Chinet et al. 1977) and for only $10-12 \%$ of thermogenesis potentiated by the administration of epinephrine (Chinet and Clausen 1984). Substrate cycling of lipids between synthesis and oxidation has been suggested to account for a portion of skeletal muscle thermogenesis in response to leptin (Solinas et al. 2004).

The mechanisms discussed above undoubtedly play some role in skeletal muscle thermogenesis. The important observation, however, is that they have not been shown to account quantitatively for all observed muscle thermogenesis, leaving room for additional mechanisms to play a role. The existence of multiple mechanisms for producing thermogenesis is 
attested to by the lack of a phenotype in many knockout mouse models. The control of temperature in homotherms is so important that multiple mechanisms have been evolved to achieve it. I propose below that the myosin ATPase activity constitutes one of the "furnaces" that produces adaptive thermogenesis in resting skeletal muscle. This proposal is supported by its great capacity for heat generation and by the fact that the structure of the thick filament is known to be modulated by physiological mechanisms that would be expected to lead to thermogenesis.

\section{The role of the SRX in thermogenesis by resting muscle}

Changes in the relative populations of the relaxed state and the SRX can lead to dramatic changes in thermogenesis in resting muscle. Two factors govern the magnitude of possible changes: (1) the concentration of myosin heads in the fibers is large, approximately $220 \mu \mathrm{M}$; (2) the difference in ATP turnover time between the two states is great. As discussed above, most myosin heads must be in the SRX in living resting muscle under basal conditions. Shifting only $20 \%$ of myosin heads from the SRX into the relaxed state would increase muscle thermogenesis by approximately a factor of two, increasing whole body metabolic rate by about $16 \%$.

There is some evidence implicating myosin in the increased thermogenesis seen during the stretch and osmotic stress discussed above. Stretch of resting frog muscle has been shown to diminish the intensity of the myosin layer lines, indicating that the myosin heads become disordered (Haselgrove 1975). Both heat output and the change in myosin layer lines did not occur for modest stretch, but did occur at larger stretches (Clinch 1968; Haselgrove 1975). Both were of long duration and were reversible when shortened (Clinch 1968; Feng 1932; Haselgrove 1975). Heat output was inhibited by hypoxia, showing metabolic control (Clinch 1968; Feng 1932). The change in the layer lines did not occur if the muscle was left overnight, showing that the changes are not simply a steric consequence of altering muscle length, again suggesting metabolic control (Haselgrove 1975). Together, these results suggest that the changes in resting thermogenesis occurring during stretch may be due to changes in thick filament structure. It would be of interest to determine if there is a wider correlation between thermogenesis and thick-filament structure under a greater variety of conditions.

The response of relaxed skeletal muscle subjected to small quick stretches is an increase in tension, known as the short-range elastic component, which has been attributed to attached cross bridges (Campbell and Lakie 1998). This response is potentiated both at long sarcomere lengths and by osmotic stress, both of which, as discussed above, also potentiate thermogenesis (Flitney 1975; Haugen and Sten-Knudsen 1981). Disordering of myosin heads is proposed here to produce greater thermogenesis and would also facilitate the interaction of myosin with actin. These interactions would be in weakly bound states that do not generate force, but contribute to stiffness. The observations described above, linking increased thick filament disorder and actomyosin interactions to conditions that lead to increased thermogenesis, provide additional support for the model of thermogenesis involving myosin.

\section{Does phosphorylation regulate the SRX in vivo?}

A strong case can be made for the potential involvement of phosphorylation of the RLC. The RLC is phosphorylated by a kinase that is activated by calcium via calmodulin (Gallagher et al. 1997). This phosphorylation does not occur to an appreciable extent during a single twitch, but does occur during a tetanus or a sustained sequence of twitches. Dephosphorylation is slow, requiring several minutes to reach baseline (Manning and Stull 1982). This system thus integrates over the recent history of activity to produce a particular level of RLC phosphorylation. RLC phosphorylation has been shown to potentiate the force of twitch contractions in fast twitch muscles (Zhi et al. 2005) and increase force at low levels of activation in permeable fibers (Persechini et al. 1985; Sweeney and Stull 1986). Phosphorylation of the RLC disorders the array of myosin heads bound to the skeletal thick filament and decreases the population of the SRX (Levine et al. 1996; Stewart et al. 2010). Disordered myosin heads would more readily interact with actin, explaining the potentiation of force at partial activation. In the model discussed here, an additional role of RLC phosphorylation is proposed. Phosphorylation potentiates force at partial levels of activation and potentiates myosin ATPase activity at even lower calcium levels, where force has been completely abolished. Both effects are due to a common action, namely, the disordering of the thick filament (Levine et al. 1996; Stewart et al. 2010). What fraction of the myosin heads are phosphorylated in human muscle during normal levels of activity? Phosphorylation levels are approximately $20-30 \%$ in both fast and slow twitch muscle fibers at rest, and these can double after a 10-s maximal contraction (Houston et al. 1987).

A mouse model lacking the calcium activated kinase that phosphorylates the RLC is not obese (Zhi et al. 2005). This is a possible indication that RLC phosphorylation plays only a little role in thermogenesis. However, multiple pathways compensate for the knockout of mechanisms known to be involved in thermogenesis, including UCP1 (Enerback et al. 1997) and sympathetic stimulation (Bachman et al. 2002). In addition, there is another kinase that produces low levels of 
RLC phosphorylation, approximately $10 \%$, in the knockout mouse (Zhi et al. 2005), and it is possible that this kinase is a mediator of thermogenesis.

A second pathway that may influence the population of the SRX is the phosphorylation of myosin binding protein-C (MBP-C). MBP-C binds to the thick filament and has been shown to affect both thick filament structure and myosin function (for review, see Oakley et al. 2007). The knockout of MBP-C in mouse cardiac muscle produces disorder of the thick filament (Colson et al. 2007; Kensler and Harris 2008). MBP-C binds to the S2 portion of myosin adjacent to the head region, and it is thought to stabilize the binding of the head back to the core of the thick filament (Ababou et al. 2008). The cardiac MBP-C has three sites for phosphorylation and can be phosphorylated by a variety of kinases, including protein kinase-A, protein kinase-C and calcium-calmodulin-dependent kinase (Oakley et al. 2007). Phosphorylation at some of these sites causes myosin heads to be released from the core of the thick filament and become disordered (Levine et al. 2001b), leading to multiple consequences for cardiac myosin function (Colson et al. 2008; Tong et al. 2008). The role of phosphorylation of MBP-C in skeletal muscle has received much less attention. While cardiac MBP-C has three phosphorylation sites, the skeletal isoform has only one (Hojlund et al. 2008; Oakley et al. 2007). One report has shown that protein kinase A can phosphorylate MBP-C from slow and fast skeletal muscles (Lim and Walsh 1986). If this phosphorylation plays a similar role as in the mouse cardiac muscle, namely, producing disordering of the thick filament array, it would also affect the population of the SRX, thereby providing a direct connection to the thermogenic effects of epinephrine, which is known to activate protein kinase A.

\section{Response to long-term overfeeding}

The response of human subjects to long-term overfeeding has shown that individuals vary widely in the amount of weight gained upon overfeeding. While some subjects put on very little weight, others will store a major fraction of the excess calories as body mass, mostly as fat (for review, see Johannsen and Ravussin 2008; Levine 2004; Schoeller 2001; Wijers et al. 2009). What process expends the ingested calories that are not stored? Measurements of the resting metabolic rate and the thermic effect of a meal have shown that neither can account for a significant fraction. Because heavy exercise was limited in many of the studies, the unexplained expenditure of energy was attributed to less strenuous activities. The largest expenditure of excess calories, and the one that has been proposed to explain much of the wide variation in weight gain, has been nonexercise activity thermogenesis, known as NEAT (Dauncey
1990; Esparza et al. 2000; Johannsen and Ravussin 2008; Levine 2004; Levine et al. 1999, 2001a; Schoeller 2001). NEAT includes any non-exercise movements and, in particular, has been proposed to depend on fidgeting. In one study, human volunteers were fed 4.2 $\mathrm{MJ}$ day $^{-1}$ excess calories for 8 weeks (Levine et al. 1999). On average, approximately half of the excess calories $\left(2.2 \mathrm{MJ}^{-1 a y}{ }^{-1}\right)$ were expended and half $\left(1.8 \mathrm{MJ} \mathrm{day}^{-1}\right)$ were stored. However, the variation in fat gain was large, varying from 0.4 to $4.2 \mathrm{~kg}$ (Fig. 6). Measurements of resting metabolic rates and the thermic effect of food showed only small changes that did not correlate with weight gain. By subtracting these two terms from total daily energy expenditure, the amount of energy expended by physical activity was determined. Because strenuous physical activity was limited, the energy expended was attributed to NEAT, which varied from -0.4 to $2.9 \mathrm{MJ}$ day $^{-1}$ (Fig. 6). The variation in NEAT was found to correlate well with that of weight gain. A number of studies have reported similar conclusions (Dauncey 1990; Esparza et al. 2000; Johannsen and Ravussin 2008; Levine 2004; Levine et al. 1999, 2001a; Schoeller 2001).

Can non-exercise muscle activity explain the large amounts of energy expended during overfeeding experiments? The efficiency of skeletal muscles is high, and it is notoriously difficult to reduce weight by exercise alone. One can estimate the energy required to perform a simple fidget, for instance, a leg swing. Let us assume that the work involves raising a $4-\mathrm{kg}$ leg by $5 \mathrm{~cm}$; the work done is approximately $2 \mathrm{~J}$. Using the muscle efficiency measured during moderate cycling, 20-30\%, (Gaesser and Brooks 1975), one fidget expends

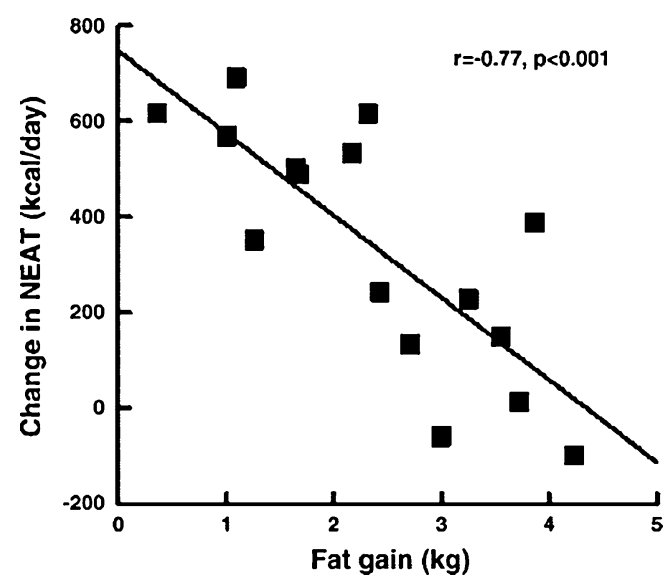

Fig. 6 Changes in non-exercise activity thermogenesis (NEAT) with overfeeding in healthy human subjects who were overfed by 4.2 $\mathrm{MJ} \mathrm{day}^{-1}$ for 8 weeks. Fat gain is plotted on the $X$-axis. Change in NEAT was calculated from NEAT values measured before and after overfeeding: NEAT $=$ total daily energy expenditure - (basic metabolic rate + thermic effect of food). The energy expended in NEAT decreases as the weight gain increases, showing that excess energy intake is approximately balanced between either NEAT or fat gain. Reproduced by permission from Levine (2004) 
approximately $6-10 \mathrm{~J}$. This is smaller than the $4 \mathrm{MJ}$ day $^{-1}$ overfeeding by more than five orders of magnitude. Although this calculation is only approximate, it is unlikely to be off by the orders of magnitude required for fidgets alone to explain the observed dissipation of calories by some subjects during overfeeding (Cooke 1999).

Despite the high efficiency of muscle, a number of lowlevel activities have been shown to expend a reasonable amount of calories (Levine et al. 2000, 2001a, 2005; Ravussin et al. 1986). There is a good correlation between energy output measured in a respiratory chamber and spontaneous activity (Dauncey 1990; Ravussin et al. 1986). Because people often engage in low-intensity exercise for a much greater length of time than they do high-intensity exercise, the former plays a larger role in energy expenditure (Johannsen and Ravussin 2008; Levine 2004). The changes in the energy expended during NEAT could arise from changes in the amount of activity or in the efficiency of performing light activities.

The model of thermogenesis by myosin described here suggests a resolution of the discrepancy between the efficiency of muscle and the energy involved in lowintensity exercise. The energy expended in a fidget or light activity is small and brief; however, the use of the muscle may lead, through phosphorylation of the RLC or through other pathways, to a more prolonged disorder of the thick filament, resulting in a more extensive heat production in the resting muscle. As described above for rabbit muscle, the transfer of only $20 \%$ of myosin heads from the SRX to the relaxed state can double resting muscle thermogenesis. Assuming that human muscle is similar to rabbit muscle, the transfer of $45 \%$ of the myosin heads during waking hours would amount to $2 \mathrm{MJ}$ day $^{-1}$, which is equal to the average energy dissipated by activity in the study by Levine et al. (1999). This level of phosphorylation is within the range measured for human muscle during moderate activities (Houston et al. 1987). This model would provide a source of thermogenesis that is additional to that required for the performance of mechanical work. If phosphorylation of the RLC is responsible for thermogenesis, heat generation would persist for several minutes following activity as that is the time course of increased phosphorylation (Manning and Stull 1982). Thus, this mechanism for thermogenesis would be expected to play a greater role during low levels of activity, where muscle spends an appreciable time in the mechanically relaxed states. This is just the type of activity that contributes to NEAT. Such a role is supported by the observation that muscle efficiency is increased in human subjects who are fasted to be $10 \%$ below normal body weight and is decreased when $10 \%$ above (Rosenbaum et al. 2003). The efficiency of walking also increases with weight loss (Foster et al. 1995). The higher efficiency at lower body weight and lower efficiency at higher body weight are both in the direction of returning to normal body weight, a known response to weight change. Of particular importance here is that the effects on efficiency are only seen at low levels of activity, $10 \mathrm{~W}$, and they disappear at higher levels. As described below, there is also a stronger genetic component to muscle efficiency at low activity levels than at high ones, which again could be easily explained by the proposed model.

Adaptive thermogenesis is controlled in part by genetic factors. The consequences to overfeeding in twins shows that there is a strong genetic component controlling the variation in weight gain, with approximately threefold more variability between pairs of twins than within pairs (Bouchard et al. 1990). There is also a genetic contribution to weight loss due to exercise (Bouchard et al. 1994). van Marken Lichtenbelt et al. (2002) found a large variation between individuals in cold-induced thermogenesis, $0.15-1.45 \mathrm{MJ} \mathrm{day}^{-1}$. The increases in thermogenesis induced by cold exposure and by overfeeding are related, showing that these two responses are regulated by common mechanisms (Wijers et al. 2007). There is also a genetic determinant of muscle efficiency. At the same level of exertion, different individuals can display different metabolic rates. Studies of identical twins have shown that at low levels of exercise the variation in metabolic rate has high heritability, $40 \%$, while for high levels of exercise the heritability disappears (Bouchard et al. 1989). The mechanism for thermogenesis presented here, which plays a more prominent role at lower levels of activity, would provide a possible framework for understanding the genetic components of adaptive thermogenesis.

The hypothesis discussed above suggests that muscle activity and its effect on myosin is tightly connected to muscle thermogenesis. Is there also an effect of thermogenesis on muscle mechanics? By analogy with another motor, active muscle is akin to a car racing down the road, relaxed muscle is similar to a car stopped at a traffic light with the motor idling and the counterpart of the SRX is a car parked beside the road with the motor off. The car at the traffic light can transition to its active counterpart very rapidly, while the parked car requires more time. Is the same true of skeletal muscle? The transition from the relaxed state to the active state would be more rapid than that from the SRX, which must enter the active state by passing through the relaxed state (Fig. 2). In fast twitch muscle, factors that increase resting thermogenesis, such as myosin phosphorylation, epinephrine and cold exposure, also lead to potentiation of twitch tensions, showing that the muscle is more easily activated (Lannergren and Westerblad 1987; Williams and Barnes 1989; Zhi et al. 2005). Thus, disordering the thick filament will lead to thermogenesis, but it also has a beneficial effect on the fiber, making it more easily activated. However, slow twitch fibers do not have potentiated twitches under the same conditions (Lannergren and Westerblad 1987; Williams and Barnes 1989; Zhi et al. 2005). This fiber difference is unexplained, 
as the SRX has also been found in slow twitch fibers (Stewart et al. 2010).

\section{The SRX as a target for possible therapeutic applications}

The exact role of the SRX has yet to be quantitatively established. However, the magnitude of possible contributions to whole body metabolic rate is great. Up-regulation of thermogenesis could be an effective mechanism for treating obesity (Wijers et al. 2009). The net difference between obesity and normal weight can arise from a small difference between daily caloric intake and dissipation, averaged over periods of years. An increase in energy usage by $0.7 \mathrm{MJ}$ day $^{-1}$ for 1 year would lead to a weight loss of $7 \mathrm{~kg}$ of fat. A pharmaceutical that transferred $10 \%$ of the myosin heads from the SRX into the relaxed state would produce this increase in thermogenesis. Thus, pharmaceuticals targeting the SRX could be extremely effective agents for the treatment of obesity.

Activation of the metabolic rate of skeletal muscle would also lead to lower blood glucose levels. Skeletal muscle is a large consumer of glucose. Following a meal, approximately one-third of the ingested glucose is stored in the liver, and insulin-mediated uptake by skeletal muscle is the destination of much of the remaining two-thirds. A major problem in type II diabetes is insulin resistance, in which insulin-mediated glucose uptake by tissues, including skeletal muscle, is impaired. It is well known that even moderate exercise can lead to decreased insulin resistance, making it an effective strategy for combating type II diabetes (Boule et al. 2003; Swartz et al. 2003). Activation of the resting metabolic rate via increased myosin ATPase activity could possibly produce effects similar to exercise. Resting muscle consumes fatty acids as a major fuel source, and up-regulation of resting metabolism would consume free fatty acids and reduce the deposition of lipid in muscle, both of which are thought to be involved in insulin resistance (Kovacs and Stumvoll 2005). In addition, ectopic expression of UCP1 in skeletal muscle increases resting energy use and also increases the metabolism of glucose (Klaus et al. 2005). Metabolic syndrome is a major and increasing problem in developed societies. The two characteristics of metabolic syndrome are obesity and type II diabetes and, as suggested above, both could be alleviated by up-regulation of the myosin ATPase activity in resting skeletal muscle.

\section{Summary}

A new state of myosin in relaxed muscle with a highly inhibited ATPase activity, the SRX, has been identified in skinned skeletal muscle fibers (Stewart et al. 2010). An obvious role of this state is to help achieve the low metabolic rate seen in resting in vivo skeletal muscle. Alterations in the population of this state have a very large capacity to alter muscle metabolism, and one physiological regulator, RLC phosphorylation, has been identified. The mechanism for muscle thermogenesis described here would operate in addition to other mechanisms that have been identified. It would function during low-level activities, which have been shown to play a major role in body weight maintenance. The identification of SRX provides a new avenue for research that links a variety of previously disparate areas, including muscle mechanics, muscle thermogenesis, diabetes and weight control.

Acknowledgments Data for this study were acquired at the Nikon Imaging Center at UCSF/QB3. The authors would like to thank Dr. Kurt Thorn and Ms. Alice Myo Thwin for their generous help in using the microscopes. The author would like to thank Dr. Edward Pate for comments on the manuscript. This work was supported by a grant from the USPHS HL32145.

Open Access This article is distributed under the terms of the Creative Commons Attribution Noncommercial License which permits any noncommercial use, distribution, and reproduction in any medium, provided the original author(s) and source are credited.

\section{References}

Ababou A, Rostkova E, Mistry S, Le Masurier C, Gautel M, Pfuhl M (2008) Myosin binding protein $\mathrm{C}$ positioned to play a key role in regulation of muscle contraction: structure and interactions of domain C1. J Mol Biol 384(3):615-630

Al-Khayat HA, Hudson L, Reedy MK, Irving TC, Squire JM (2003) Myosin head configuration in relaxed insect flight muscle: x-ray modeled resting cross-bridges in a pre-powerstroke state are poised for actin binding. Biophys J 85(2):1063-1079

Al-Khayat HA, Morris EP, Squire JM (2009) The 7-stranded structure of relaxed scallop muscle myosin filaments: support for a common head configuration in myosin-regulated muscles. J Struct Biol 166(2):183-194

Alamo L, Wriggers W, Pinto A, Bartoli F, Salazar L, Zhao FQ, Craig R, Padron R (2008) Three-dimensional reconstruction of tarantula myosin filaments suggests how phosphorylation may regulate myosin activity. J Mol Biol 384(4):780-797

Ankrett RJ, Rowe AJ, Cross RA, Kendrick-Jones J, Bagshaw CR (1991) A folded (10 S) conformer of myosin from a striated muscle and its implications for regulation of ATPase activity. $\mathrm{J}$ Mol Biol 217(2):323-335

Astrup A, Bulow J, Christensen NJ, Madsen J, Quaade F (1986) Facultative thermogenesis induced by carbohydrate: a skeletal muscle component mediated by epinephrine. Am J Physiol 250(2 Pt 1):E226-E229

Astrup A, Simonsen L, Bulow J, Madsen J, Christensen NJ (1989) Epinephrine mediates facultative carbohydrate-induced thermogenesis in human skeletal muscle. Am J Physiol 257(3 Pt 1): E340-E345

Bachman ES, Dhillon H, Zhang CY, Cinti S, Bianco AC, Kobilka BK, Lowell BB (2002) betaAR signaling required for diet-induced 
thermogenesis and obesity resistance. Science 297(5582):843845

Barclay CJ, Woledge RC, Curtin NA (2009) Effects of UCP3 genotype, temperature and muscle type on energy turnover of resting mouse skeletal muscle. Pflugers Arch 457(4):857-864

Bouchard C, Tremblay A, Nadeau A, Despres JP, Theriault G, Boulay MR, Lortie G, Leblanc C, Fournier G (1989) Genetic effect in resting and exercise metabolic rates. Metabolism 38 (4):364-370

Bouchard C, Tremblay A, Despres JP, Nadeau A, Lupien PJ, Theriault G, Dussault J, Moorjani S, Pinault S, Fournier G (1990) The response to long-term overfeeding in identical twins. $\mathrm{N}$ Engl $\mathrm{J}$ Med 322(21):1477-1482

Bouchard C, Tremblay A, Despres JP, Theriault G, Nadeau A, Lupien PJ, Moorjani S, Prudhomme D, Fournier G (1994) The response to exercise with constant energy intake in identical twins. Obes Res 2(5):400-410

Boule NG, Kenny GP, Haddad E, Wells GA, Sigal RJ (2003) Metaanalysis of the effect of structured exercise training on cardiorespiratory fitness in Type 2 diabetes mellitus. Diabetologia 46(8):1071-1081

Campbell KS, Lakie M (1998) A cross-bridge mechanism can explain the thixotropic short-range elastic component of relaxed frog skeletal muscle. J Physiol 510(Pt 3):941-962

Chinet A (1993) $\mathrm{Ca}(2+)$-dependent heat production by rat skeletal muscle in hypertonic media depends on $\mathrm{Na}(+)-\mathrm{Cl}-$ co-transport stimulation. J Physiol 461:689-703

Chinet A, Clausen T (1984) Energetics of active sodium-potassium transport following stimulation with insulin, adrenaline or salbutamol in rat soleus muscle. Pflugers Arch 401(2):160-166

Chinet A, Clausen T, Girardier L (1977) Microcalorimetric determination of energy expenditure due to active sodiumpotassium transport in the soleus muscle and brown adipose tissue of the rat. J Physiol 265(1):43-61

Chinet A, Decrouy A, Even PC (1992) $\mathrm{Ca}(2+)$-dependent heat production under basal and near-basal conditions in the mouse soleus muscle. J Physiol 455:663-678

Clinch NF (1968) On the increase in rate of heat production caused by stretch in frog's skeletal muscle. J Physiol 196(2):397-414

Colson BA, Bekyarova T, Fitzsimons DP, Irving TC, Moss RL (2007) Radial displacement of myosin cross-bridges in mouse myocardium due to ablation of myosin binding protein-C. J Mol Biol 367(1):36-41

Colson BA, Bekyarova T, Locher MR, Fitzsimons DP, Irving TC, Moss RL (2008) Protein kinase A-mediated phosphorylation of cMyBP-C increases proximity of myosin heads to actin in resting myocardium. Circ Res 103(3):244-251

Cooke R (1999) A fidgeters calculation. Science 284:1125

Cooke R, Pate E (1985) The effects of ADP and phosphate on the contraction of muscle fibers. Biophys J 48(5):789-798

Costford SR, Seifert EL, Bezaire V, Gerritz M, Bevilacqua L, Gowing A, Harper ME (2007) The energetic implications of uncoupling protein-3 in skeletal muscle. Appl Physiol Nutr Metab 32 (5):884-894

Craig R, Woodhead JL (2006) Structure and function of myosin filaments. Curr Opin Struct Biol 16(2):204-212

Cremo CR, Neuron JM, Yount RG (1990) Interaction of myosin subfragment 1 with fluorescent ribose-modified nucleotides. A comparison of vanadate trapping and SH1-SH2 cross-linking. Biochemistry 29(13):3309-3319

Cross RA, Jackson AP, Citi S, Kendrick-Jones J, Bagshaw CR (1988) Active site trapping of nucleotide by smooth and non-muscle myosins. J Mol Biol 203(1):173-181

Dauncey MJ (1981) Influence of mild cold on $24 \mathrm{~h}$ energy expenditure, resting metabolism and diet-induced thermogenesis. Br J Nutr 45(2):257-267
Dauncey MJ (1990) Activity and energy expenditure. Can J Physiol Pharmacol 68(1):17-27

Duchamp C, Barre H (1993) Skeletal muscle as the major site of nonshivering thermogenesis in cold-acclimated ducklings. Am J Physiol 265(5 Pt 2):R1076-R1083

Dulloo AG, Decrouy A, Chinet A (1994) Suppression of $\mathrm{Ca}(2+)-$ dependent heat production in mouse skeletal muscle by high fish oil consumption. Metabolism 43(8):931-934

Dulloo AG, Stock MJ, Solinas G, Boss O, Montani JP, Seydoux J (2002) Leptin directly stimulates thermogenesis in skeletal muscle. FEBS Lett 515(1-3):109-113

Enerback S, Jacobsson A, Simpson EM, Guerra C, Yamashita H, Harper ME, Kozak LP (1997) Mice lacking mitochondrial uncoupling protein are cold-sensitive but not obese. Nature 387(6628):90-94

Esparza J, Fox C, Harper IT, Bennett PH, Schulz LO, Valencia ME, Ravussin E (2000) Daily energy expenditure in Mexican and USA Pima indians: low physical activity as a possible cause of obesity. Int J Obes Relat Metab Disord 24(1):55-59

Feng TP (1932) The effect of length on the resting metabolism of muscle. J Physiol 74(4):441-454

Ferenczi MA, Homsher E, Simmons RM, Trentham DR (1978) Reaction mechanism of the magnesium ion-dependent adenosine triphosphatase of frog muscle myosin and subfragment 1. Biochem $\mathrm{J}$ 171(1): 165-175

Flitney FW (1975) Light scattering associated with tension changes in the short-range elastic component of resting frog's muscle. J Physiol 244(1):1-14

Foster GD, Wadden TA, Kendrick ZV, Letizia KA, Lander DP, Conill AM (1995) The energy cost of walking before and after significant weight loss. Med Sci Sports Exerc 27(6):888-894

Gaesser GA, Brooks GA (1975) Muscular efficiency during steadyrate exercise: effects of speed and work rate. J Appl Physiol 38 (6):1132-1139

Gallagher PJ, Herring BP, Stull JT (1997) Myosin light chain kinases. J Muscle Res Cell Motil 18(1):1-16

Gong DW, Monemdjou S, Gavrilova O, Leon LR, Marcus-Samuels B, Chou CJ, Everett C, Kozak LP, Li C, Deng C, Harper ME, Reitman ML (2000) Lack of obesity and normal response to fasting and thyroid hormone in mice lacking uncoupling protein-3. J Biol Chem 275(21):16251-16257

Gutierrez G, Pohil RJ, Narayana P (1989) Skeletal muscle O2 consumption and energy metabolism during hypoxemia. J Appl Physiol 66(5):2117-2123

Hackney DD, Stock MF (2008) Kinesin tail domains and Mg2+ directly inhibit release of ADP from head domains in the absence of microtubules. Biochemistry 47(29):7770-7778

Haselgrove JC (1975) X-ray evidence for conformational changes in the myosin filaments of vertebrate striated muscle. J Mol Biol 92 (1):113-143

Haugen P, Sten-Knudsen O (1981) The dependence of the short-range elasticity on sarcomere length in resting isolated frog muscle fibres. Acta Physiol Scand 112(2):113-120

Hojlund K, Yi Z, Hwang H, Bowen B, Lefort N, Flynn CR, Langlais P, Weintraub ST, Mandarino LJ (2008) Characterization of the human skeletal muscle proteome by one-dimensional gel electrophoresis and HPLC-ESI-MS/MS. Mol Cell Proteomics 7(2):257-267

Houston ME, Lingley MD, Stuart DS, Grange RW (1987) Myosin light chain phosphorylation in intact human muscle. FEBS Lett 219(2):469-471

Huxley HE, Brown W, Holmes KC (1965) Constancy of axial spacings in frog sartorius muscle during contraction. Nature 206(991): 1358

Johannsen DL, Ravussin E (2008) Spontaneous physical activity: relationship between fidgeting and body weight control. Curr Opin Endocrinol Diabetes Obes 15(5):409-415 
Jung HS, Komatsu S, Ikebe M, Craig R (2008) Head-head and headtail interaction: a general mechanism for switching off myosin II activity in cells. Mol Biol Cell 19(8):3234-3242

Kensler RW, Harris SP (2008) The structure of isolated cardiac Myosin thick filaments from cardiac Myosin binding protein-C knockout mice. Biophys J 94(5):1707-1718

Kensler RW, Stewart M (1993) The relaxed crossbridge pattern in isolated rabbit psoas muscle thick filaments. J Cell Sci 105(Pt 3):841-848

Klaus S, Rudolph B, Dohrmann C, Wehr R (2005) Expression of uncoupling protein 1 in skeletal muscle decreases muscle energy efficiency and affects thermoregulation and substrate oxidation. Physiol Genomics 21(2):193-200

Kovacs P, Stumvoll M (2005) Fatty acids and insulin resistance in muscle and liver. Best Pract Res Clin Endocrinol Metab 19(4):625-635

Kushmerick MJ, Paul RJ (1976) Aerobic recovery metabolism following a single isometric tetanus in frog sartorius muscle at $0^{\circ} \mathrm{C}$. J Physiol:693-715

Lannergren J, Westerblad H (1987) The temperature dependence of isometric contractions of single, intact fibres dissected from a mouse foot muscle. J Physiol 390:285-293

Levine JA (2004) Nonexercise activity thermogenesis (NEAT): environment and biology. Am J Physiol Endocrinol Metab 286 (5):E675-E685

Levine RJ, Kensler RW, Yang Z, Stull JT, Sweeney HL (1996) Myosin light chain phosphorylation affects the structure of rabbit skeletal muscle thick filaments. Biophys J 71(2):898-907

Levine JA, Eberhardt NL, Jensen MD (1999) Role of nonexercise activity thermogenesis in resistance to fat gain in humans. Science 283(5399):212-214

Levine JA, Schleusner SJ, Jensen MD (2000) Energy expenditure of nonexercise activity. Am J Clin Nutr 72(6):1451-1454

Levine J, Melanson EL, Westerterp KR, Hill JO (2001a) Measurement of the components of nonexercise activity thermogenesis. Am J Physiol Endocrinol Metab 281(4):E670-E675

Levine R, Weisberg A, Kulikovskaya I, McClellan G, Winegrad S (2001b) Multiple structures of thick filaments in resting cardiac muscle and their influence on cross-bridge interactions. Biophys J 81(2):1070-1082

Levine JA, Lanningham-Foster LM, McCrady SK, Krizan AC, Olson LR, Kane PH, Jensen MD, Clark MM (2005) Interindividual variation in posture allocation: possible role in human obesity. Science 307(5709):584-586

Lim MS, Walsh MP (1986) Phosphorylation of skeletal and cardiac muscle C-proteins by the catalytic subunit of cAMP-dependent protein kinase. Biochem Cell Biol 64(7):622-630

Lowell BB, Spiegelman BM (2000) Towards a molecular understanding of adaptive thermogenesis. Nature 404(6778):652-660

Manning DR, Stull JT (1982) Myosin light chain phosphorylationdephosphorylation in mammalian skeletal muscle. Am J Physiol 242(3):C234-C241

Myburgh KH, Franks-Skiba K, Cooke R (1995) Nucleotide turnover rate measured in fully relaxed rabbit skeletal muscle myofibrils. J Gen Physiol 106(5):957-973

Oakley CE, Chamoun J, Brown LJ, Hambly BD (2007) Myosin binding protein-C: enigmatic regulator of cardiac contraction. Int J Biochem Cell Biol 39(12):2161-2166

Paolone VJ, Paolone AM (1995) Thermogenesis during rest and exercise in cold air. Can J Physiol Pharmacol 73(8):1149-1153

Persechini A, Stull JT, Cooke R (1985) The effect of myosin phosphorylation on the contractile properties of skinned rabbit skeletal muscle fibers. J Biol Chem 260(13):7951-7954

Ravussin E, Lillioja S, Anderson TE, Christin L, Bogardus C (1986) Determinants of 24-hour energy expenditure in man. Methods and results using a respiratory chamber. J Clin Invest 78 (6): $1568-1578$
Reedy MK, Lucaveche C, Reedy MC, Taylor KA, Somasundaram B (1992) Hybrid sacomeres of insect flight muscle, where rigor overlap zones abut relaxed H-bands. Biophys J 61:A286

Rosenbaum M, Vandenborne K, Goldsmith R, Simoneau JA, Heymsfield S, Joanisse DR, Hirsch J, Murphy E, Matthews D, Segal KR, Leibel RL (2003) Effects of experimental weight perturbation on skeletal muscle work efficiency in human subjects. Am J Physiol Regul Integr Comp Physiol 285(1):R183-R192

Schoeller DA (2001) The importance of clinical research: the role of thermogenesis in human obesity. Am J Clin Nutr 73(3):511-516

Simeckova M, Jansky L, Lesna II, Vybiral S, Sramek P (2000) Role of beta adrenoceptors in metabolic and cardiovascular responses of cold exposed humans. J Therm Biol 25(6):437-442

Simonsen L, Bulow J, Madsen J, Christensen NJ (1992) Thermogenic response to epinephrine in the forearm and abdominal subcutaneous adipose tissue. Am J Physiol 263(5 Pt 1):E850-E855

Sims EA, Danforth E Jr (1987) Expenditure and storage of energy in man. J Clin Invest 79(4):1019-1025

Solandt DY (1936) The effect of potassium on the excitability and resting metabolism of frog's muscle. J Physiol 86(2):162-170

Solinas G, Summermatter S, Mainieri D, Gubler M, Pirola L, Wymann MP, Rusconi S, Montani JP, Seydoux J, Dulloo AG (2004) The direct effect of leptin on skeletal muscle thermogenesis is mediated by substrate cycling between de novo lipogenesis and lipid oxidation. FEBS Lett 577(3):539-544

Stewart MA, Franks-Skiba K, Chen S, Cooke R (2010) Myosin ATP turnover rate is a mechanism involved in thermogenesis in resting skeletal muscle fibers. Proc Natl Acad Sci USA 107:430-435

Swartz AM, Strath SJ, Bassett DR, Moore JB, Redwine BA, Groer M, Thompson DL (2003) Increasing daily walking improves glucose tolerance in overweight women. Prev Med 37(4):356362

Sweeney HL, Stull JT (1986) Phosphorylation of myosin in permeabilized mammalian cardiac and skeletal muscle cells. Am J Physiol 250(4 Pt 1):C657-C660

Tong CW, Stelzer JE, Greaser ML, Powers PA, Moss RL (2008) Acceleration of crossbridge kinetics by protein kinase $\mathrm{A}$ phosphorylation of cardiac myosin binding protein $\mathrm{C}$ modulates cardiac function. Circ Res 103(9):974-982

van Marken Lichtenbelt WD, Daanen HA (2003) Cold-induced metabolism. Curr Opin Clin Nutr Metab Care 6(4):469-475

van Marken Lichtenbelt WD, Schrauwen P, van De Kerckhove S, Westerterp-Plantenga MS (2002) Individual variation in body temperature and energy expenditure in response to mild cold. Am J Physiol Endocrinol Metab 282(5):E1077-E1083

Vibert P, Craig R (1985) Structural changes that occur in scallop myosin filaments upon activation. J Cell Biol 101(3):830837

Vidal-Puig AJ, Grujic D, Zhang CY, Hagen T, Boss O, Ido Y, Szczepanik A, Wade J, Mootha V, Cortright R, Muoio DM, Lowell BB (2000) Energy metabolism in uncoupling protein 3 gene knockout mice. J Biol Chem 275(21):16258-16266

Vinnakota KC, Rusk J, Palmer L, Shankland E, Kushmerick MJ (2010) Common phenotype of resting mouse extensor digitorum longus and soleus muscles: equal ATPase and glycolytic flux during transient anoxia. J Physiol 588(Pt 11):1961-1983

Wendt T, Taylor D, Messier T, Trybus KM, Taylor KA (1999) Visualization of head-head interactions in the inhibited state of smooth muscle myosin. J Cell Biol 147(7):1385-1390

West TG, Boutilier RG (1998) Metabolic suppression in anoxic frog muscle. J Comp Physiol B 168(4):273-280

Wijers SL, Saris WH, van Marken Lichtenbelt WD (2007) Individual thermogenic responses to mild cold and overfeeding are closely related. J Clin Endocrinol Metab 92(11):4299-4305 
Wijers SL, Saris WH, van Marken Lichtenbelt WD (2009) Recent advances in adaptive thermogenesis: potential implications for the treatment of obesity. Obes Rev 10(2):218-226

Williams JH, Barnes WS (1989) The positive inotropic effect of epinephrine on skeletal muscle: a brief review. Muscle Nerve 12 (12):968-975

Woodhead JL, Zhao FQ, Craig R, Egelman EH, Alamo L, Padron R (2005) Atomic model of a myosin filament in the relaxed state. Nature 436(7054):1195-1199

Woodward SK, Eccleston JF, Geeves MA (1991) Kinetics of the interaction of $2^{\prime}\left(3^{\prime}\right)-O-(N$-methylanthraniloyl)-ATP with myosin subfragment 1 and actomyosin subfragment 1: characterization of two acto-S1-ADP complexes. Biochemistry 30 (2):422-430

Xu S, Offer G, Gu J, White HD, Yu LC (2003) Temperature and ligand dependence of conformation and helical order in myosin filaments. Biochemistry 42(2):390-401
Yamada K (1970) The increase in the rate of heat production of frog's skeletal muscle caused by hypertonic solutions. J Physiol 208 (1):49-64

Zhao FQ, Craig R, Woodhead JL (2009) Head-head interaction characterizes the relaxed state of Limulus muscle myosin filaments. J Mol Biol 385(2):423-431

Zhi G, Ryder JW, Huang J, Ding P, Chen Y, Zhao Y, Kamm KE, Stull JT (2005) Myosin light chain kinase and myosin phosphorylation effect frequency-dependent potentiation of skeletal muscle contraction. Proc Natl Acad Sci USA 102 (48):17519-17524

Zoghbi ME, Woodhead JL, Moss RL, Craig R (2008) Threedimensional structure of vertebrate cardiac muscle myosin filaments. Proc Natl Acad Sci USA 105(7):2386-2390

Zurlo F, Larson K, Bogardus C, Ravussin E (1990) Skeletal muscle metabolism is a major determinant of resting energy expenditure. J Clin Invest 86(5):1423-1427 